\title{
A MODEL FOR STUDIES OF THE DEFORMABLE RIB CAGE
}

\author{
Robert F. Closkey, Albert B. Schultz and Carl W. Luchies \\ University of Michigan, Department of Mechanical Engineering and Applied Mechanics, Ann Arbor, \\ MI 48109-2125, U.S.A.
}

\begin{abstract}
An earlier model for the study of rib cage mechanics was modified so that rib deformity in scoliosis could be better represented. The rigid ribs of that model were replaced by five-segment deformable ribs. Literature data on cadaver rib mechanical behavior were used to assign stiffnesses to the new individual model ribs so that experimental and model rib deflections agreed. Shear and tension/compression stiffnesses had little effect on individual rib deformation, but bending stiffinesses had a major effect. Level-to-level differences in mechanical behavior could be explained almost exclusively by level to level differences in the rib shape. The model ribs were then assembled into a whole rib cage. Computer simulations of whole rib cage behaviors, both in vivo and in vitro, showed a reasonable agreement with the measured behaviors.

The model was used to study rib cage mechanics in two scolioses, one with a $43^{\circ}$ and the other with a $70^{\circ}$ Cobb angle. Scoliotic rib cage deformities were quantified by parameters measuring the rib cage lateral offset, rib cage axial rotation, rib cage volume and rib distortion. Rib distortion was quantified both in bestfit and simulated computer tomography (CT) scan planes. Model rib distortion was much smaller in best-fit planes than in CT planes. The total rib cage volume changed little in the presence of the scolioses, but it became asymmetrically distributed.
\end{abstract}

\section{INTRODUCTION}

Although rib cage deformities that occur in scoliosis are often cosmetically displeasing and sometimes compromise cardiopulmonary function enough to be life-threatening, little is understood regarding the biomechanics of these deformities. The reduction of pulmonary function in scoliosis has been documented (Aaro and Ohlund, 1984; Gazioglu et al., 1968; Pecorelli et al., 1983; Winter et al., 1975; Smith et al., 1991) but the role of rib cage involvement in this reduction remains unclear. Surgical techniques that correct a lateral curve in the spine alter both cosmesis (Aaro and Dahlborn, 1981, 1982; Gaines et al., 1981; Jefferson et al., 1988; Thulbourne and Gillespie, 1976; Weatherly et al., 1987) and pulmonary function (Kumano and Tsuyama, 1985; Lindh and Bjure, 1975; Ogilvie and Schendel, 1988), but it is unclear how spine configuration corrections change rib cage configuration.

To foster investigations of rib cage deformities in scoliosis, we constructed a biomechanical model of a rib cage that allowed individual model ribs to deform. Our study addressed a number of issues. (1) What stiffnesses of the deformable rib elements are needed to make model individual rib behavior agree with real rib behavior? (2) If model and real individual rib behaviors agree, do model and real overall cage behaviors also agree in structurally normal trunks? (3) What are suitable quantitative measures of rib cage deformities in scoliosis? (4) What is the effect of rib cage deformity in scoliosis on cage volume change? (5) What distortions arise in model ribs when the spine is changed from a structurally normal configuration to

Received in final form 16 September 1991. configurations typical of scoliosis, and how might these distortions relate to distortions apparent in computer tomography (CT) scans of cage cross sections?

\section{METHODS}

\section{Model construction}

General. A previously reported biomechanical model of the thoracolumbar spine, sacrum, sternum and rib cage (Andriacchi et al., 1974) represented the bony structure of the trunk by a collection of rigid bodies and the soft tissue connecting them by a collection of deformable spring and beam elements. Model response to imposed forces and/or displacements was computed using the direct stiffness method of engineering structural analysis. Our model replaces the 20 rigid ribs of the earlier model with deformable ribs (Fig. 1). The floating ribs were not included in the model of Andriacchi et al. (1974), nor have they been included in our model, as they do not seem to have a major effect on rib cage overall mechanics. The deformable ribs have each been represented by five cylindrical rigid bodies interconnected by four beam elements. This modification increased the number of rigid bodies from 39 in the earlier model to 119 in our model, the number of beam elements from 59 to 139 , and the number of spring elements from 176 to 344 . The total model degrees-of-freedom is increased from 234 to 714.

Geometry. The eight points used by Andriacchi et al. (1974) to define the geometry of each rigid rib were also used to locate the five cylindrical bodies, so that the geometry of the 1974 model has been retained in essence (Fig. 1). 


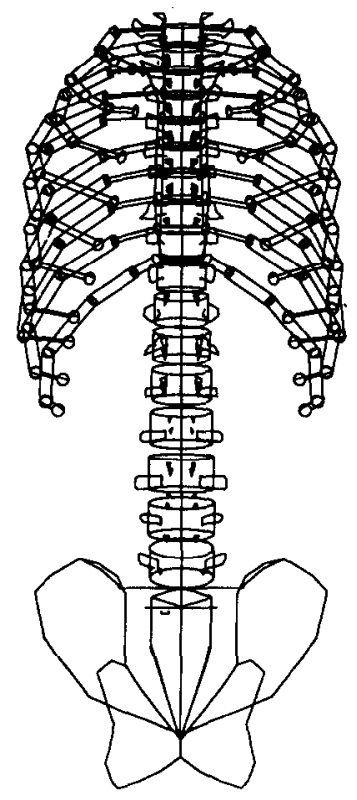

(a)

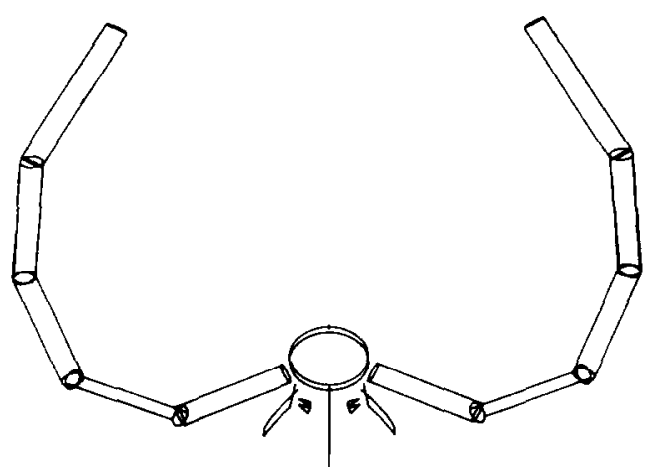

(b)

Fig. 1. (a) Schematic representation of the rib cage in its undeformed configuration. (b) Superior view showing how a rib is represented by five cylindrical rigid bodies.

Stiffness properties. The deformable elements representing the costosternal and costovertebral tissues are the same as those used in the 1974 model as are the stiffnesses of the deformable elements interconnecting the vertebrae. However, the representation of the intercostal ligamentous tissues has been modified from that of the 1974 model. Each of the five cylinders of each rib was connected to its superiorly and inferiorly adjacent rib cylinder using a spring element. Thus, the intercostal connections earlier modeled by two springs per rib level are now represented by 20 springs per level, evenly spaced along the length of the ribs. The stiffnesses of these elements were adjusted to account for this redistribution.

Six additional springs, four to represent the pelvosternal ligamentous tissue ties and two to represent ligamentous ties to the clavicles, have been included in our model. The pelvosternal springs connected the superior portion of the pelvis to the inferior portion of the sternum. The clavicular springs connected the superior portion of the sternum to the $\mathrm{T} 1$ vertebra on both sides of the spine. These additional ties represent the sternal support that exists anatomically (Feiss, 1906).

Stiffness values were assigned to the beam elements interconnecting the cylinders of each rib using the data of Schultz et al. (1974). They reported deflections of cadaver ribs from levels $2,4,6,8$ and 10 in response to $7.4 \mathrm{~N}(0.75 \mathrm{kP})$ loads. They applied these loads successively in mutually orthogonal directions to the free anterior ends of individual ribs with fixed posterior ends. A Cartesian coordinate system local to each isolated model rib corresponding to the system used by Schultz et al. (1974) was created. The anterior/posterior loading direction of a rib was defined as being along the line joining the point closest to the vertebral body center of the cylinder closest to the vertebra with the anteriormost point of the cylinder closest to the sternum. The inferior/superior line of action was perpendicular to the best-fit plane of the rib. The medial/lateral loading direction was defined by the cross product of the other two direction vectors.

Each beam element had six unknown stiffness values: two bending, two shear, one axial and one torsional. Axial and shear relative displacements between adjacent sections of bony structures are small. Therefore, the translational axial and the two shear stiffnesses of the beam elements were assigned values large enough to prevent observable translation in these directions. To verify that these translational stiffness values mattered little, simulations were run with these stiffnesses decreased by a factor of 100 and the results of the two sets of simulations were compared.

This left only the three bending stiffnesses unknown. It was tentatively assumed that all 80 beam elements could be assigned the same set of bending stiffnesses. The vertebral ends of the model ribs were fixed and the sternal ends were loaded anteriorly, posteriorly, superiorly, inferiorly, medially and laterally with $7.4 \mathrm{~N}$ forces, as done by Schultz et al. (1974). The three unknown bending stiffnesses were adjusted as described below until an acceptable level of agreement 
between simulated and experimental rib defiections in all loading directions was obtained (Results, Fig. 5). This produced deflections at all five rib levels in satisfactory agreement with those experimentally measured; so there was no need to alter the tentative assumption.

The lateral-bending stiffness controlled primarily the anterior/posterior and medial/lateral deflections of the rib. During simulated loadings in these directions, this stiffness was adjusted until model deflections were in reasonable agreement with experimental deflections in both directions. The torsional and flexion/extension stiffnesses of the beam elements were first determined during simulations of superior/inferior pulls. The torsional stiffness and then the flexion/ extension stiffness was readjusted until the predicted superior/inferior deflections agreed with the experimental values, while retaining the agreement during pull simulations in the other directions.

The properties assigned to the springs representing the abdominal and clavicular connections were initially calculated from the stiffness data reported by Yamada (1970). These stiffness values were then adjusted through model simulations, described in the next section; so the behavior of the assembled model rib cage agreed with whole rib cage behavior found in experiments performed on human subjects.

Calculation of rib cage volume. The total volume of the model rib cage was defined as that of a polyhedron bounded circumferentially at each level by the model ribs, and superiorly and inferiorly by the surfaces defined by Rib 1 and Rib 10. The effect of diaphragm configuration was not considered. The volume was thus defined by 140 points, 14 per rib level (Fig. 2). The volume between every two rib levels was divided into twelve wedge-shaped solids. Each wedge was defined by six points; three points from superior-level nodes and three points from the corresponding nodes of its inferiorly adjacent neighbor. Each wedge was further decomposed into three tetrahedra and the volume of

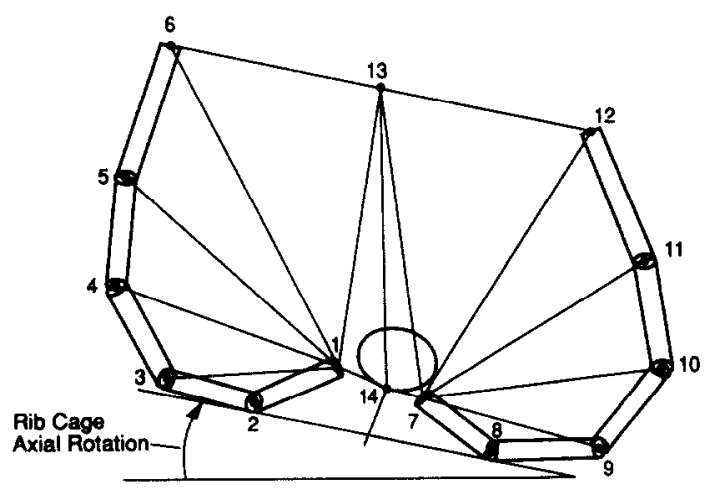

Fig. 2. Superior view of a deformed rib, showing the 14 points that define the 12 wedge sections between rib levels. These wedge sections are used to calculate the rib cage volume. The definition of rib cage axial rotation is also shown. each tetrahedron was computed. The three tetrahedra volumes yielded the wedge volume, the 12 wedges yielded the volume between rib levels, and the nine inter-rib levels yielded the total rib cage volume. The locations of the 140 points used to calculate the rib cage volume were chosen to be bilaterally symmetric in the structurally normal state so that convex and concave side volumes could be determined in the deformed states of scoliosis.

Validation studies - behavior of assembled rib cage. Three experimental investigations reported in the literature were simulated to evaluate the agreement of the predicted assembled rib cage deflections with the deflections observed in humans. Agostoni et al. (1966) reported the anterior/posterior and lateral rib cage deflections in supine male volunteers when a lateral-squeezing load was applied to their rib cage. To simulate these experiments, the sacrum of the model was fixed and the posterior most portion of the rib cage was prevented from moving to represent the constraints imposed by the surface supporting the subjects. A $100 \mathrm{~N}$ lateral squeezing load was applied to the ribs at the xiphoid process level. This load was evenly distributed over rib-pairs 6-10. Relative displacements between the sternum and vertebrae at the xiphoid level as measured by Agostoni et al. (1966) were compared to the change in the anterior/posterior rib cage diameter predicted by the model. Lateraldiameter changes were compared similarly.

Patrick et al. (1965) and Nahum et al. (1970) examined deflections of cadaver rib cages in response to sternal compressive loads applied to upright cadavers whose backs were supported. To simulate these conditions in the model, the sacrum was fixed and the posteriormost points on the ribs were prevented from moving. A $100 \mathrm{~N}$ compressive load was applied to the sternum just superior to the xiphoid process. The resulting deflections of the sternum were then noted and compared to the experimental values.

To evaluate the effect of rib cage support of the spine in axial rotations, Lovett (1905) fixed the sacrum of a cadaver and axially rotated its $T 1$ vertebra by $51^{\circ}$. $\mathrm{He}$ reported the resulting rotations from $\mathrm{T} 1$ to $\mathrm{L} 5$. To simulate this experiment the sacrum was fixed and a $51^{\circ}$ axial rotation was imposed on the $T 1$ vertebra. The rotations of the other vertebrae in response to the rotation at $\mathrm{T} 1$ were then noted from $\mathrm{T} 2$ to $\mathrm{L} 5$ levels and compared with Lovett's findings. No more comprehensive or more recent comparable experimental data could be found.

\section{Imposition of scoliosis}

To examine the usefulness of this model in investigating rib deformities in scoliosis, a mild $43^{\circ}$ Cobb angle mid-thoracic curve and a more severe $70^{\circ}$ mid-thoracic curve (Table 2) were modeled by prescribing the known configurations of all vertebrae in these curves to the model vertebrae. The pelvis of the 
normal spine was fixed, while the rib cage and sternum were left to freely deform in response to the scoliosis. The resulting rib cage deformations were then calculated, quantified as described below, and compared to clinical data on rib deformity.

\section{Measures of scoliotic rib cage deformity}

Rib cage axial rotation. Rib cage axial rotation was defined, following Dansereau and Stokes (1988) and Stokes (1989), as the angle between a frontal plane and the tangent to the rib cage at the apical vertebral level (Fig. 2).

Rib cage lateral offset. Rib cage lateral offset was defined as the lateral distance between the midline of the sacrum and the geometric center of the deformed rib cage, midway transversely between the lateralmost points of the cage (Fig. 3).

Rib distortion in the apical CT scan plane and apical rib best-fit plane. Rib distortion in the apical CT scan plane was determined by locating segments of ribs which passed through a $1 \mathrm{~cm}$ thick transverse slice at the apical spine level. The points on the longitudinal axis of each model rib cylinder contained in that slice at $0.1 \mathrm{~cm}$ intervals were collected. Thus, if a cylinder

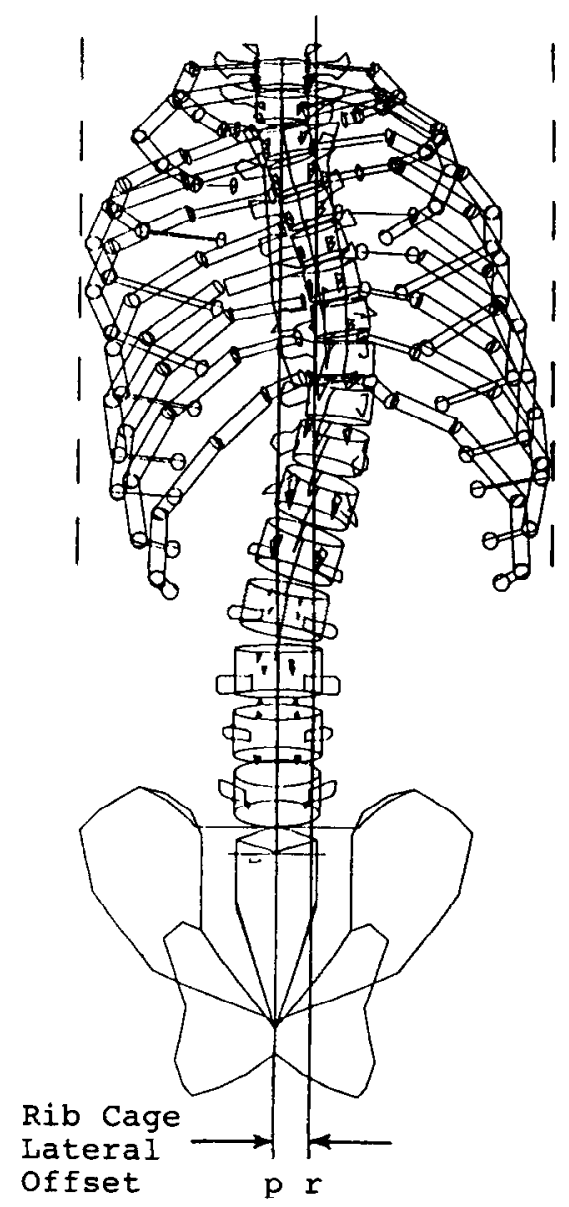

Fig. 3. Definition of rib cage lateral offset (p-center of pelvis; $r$ - center of rib cage). was completely contained within the $1 \mathrm{~cm}$ slice, ten points from that cylinder were used. Once all 20 ribs were scanned for points to be included, the included points were plotted to obtain a CT plane composite 'rib' [Fig. 4(a)].

The apical rib best-fit (least squares) plane was found and the nodes of the apical rib were projected onto that plane. Radii of curvature were then established for the posterior portions of both left- and rightprojected and CT composite ribs (Fig. 4). Rib distortion was defined as the concave rib radius minus the convex rib radius divided by the larger radius. Rib radius calculation was straightforward for the leastsquares projection. The radii were those of the two circles defined by the three posterior-most points of the right and left rib. The CT plane composite rib often contained over 90 points, but they formed distinct groups. A least-squares circle was fit to all points in the three posterior-most groups.

\section{RESULTS}

\section{Individual rib behavior}

The stiffness values ultimately assigned to all beam elements interconnecting the rib cylinders were an axial stiffness of $100 \mathrm{kN} \mathrm{cm}^{-1}$, two shear stiffnesses of $100 \mathrm{kN} \mathrm{cm}^{-1}$, an anterior/posterior bending stiffness of $2 \mathrm{kN} \mathrm{cm} \mathrm{rad}^{-1}$, a superior/inferior bending stiffness of $1.8 \mathrm{kN} \mathrm{cm} \mathrm{rad}-1$ and a torsional stiffness of $3 \mathrm{kN} \mathrm{cm} \mathrm{rad}{ }^{-1}$ (Table 1).

Nearly all model individual rib deflections were within two standard deviations and within $0.5 \mathrm{~cm}$ of the mean experimental deflections under $7.4 \mathrm{~N}$ forces (Fig. 5). Exceptions occurred in inferior loading of rib 2 and anterior loading of ribs 4 and 6 . In these cases the experimental deflections for one direction of force application were different from those for the opposite direction. The assumption of linearity in model behavior prevented the occurrence of such differences in the model. Despite this, agreement of the model and measured individual rib deflections in these three cases was within $1.3 \mathrm{~cm}$.

The axial stiffness and the two shear stiffnesses of the rib beam elements were found, as assumed, to have little influence on rib deflection. When these three translational stiffnesses were decreased by two orders of magnitude, only small changes in rib deflection occurred, no matter what the level or the applied force direction was. For example, the deflection of rib 8 under a medial load increased from 3.46 to $3.51 \mathrm{~cm}$ with the two orders of magnitude decrease in these three stiffnesses.

\section{Assembled rib cage behavior}

In simulations of all three experiments regarding the behavior of the assembled rib cage, a reasonable agreement between model predictions and experimental measurements was found (Figs 6-8). The response of the present model to an evenly distributed 


\section{ANTERIOR}

a) Rib Distortion $=0.08$

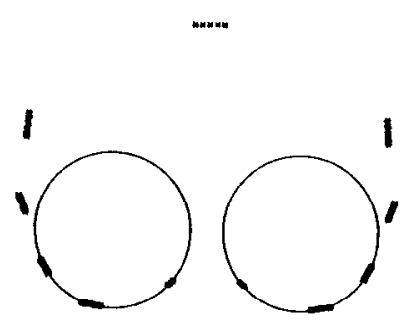

b) Rib Distortion $=11.48$

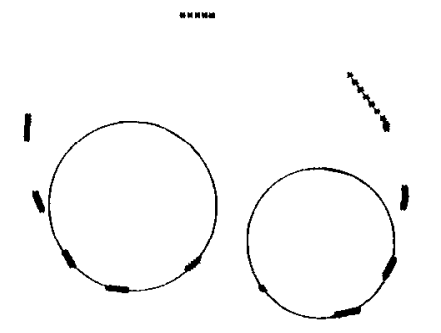

c) Rib Distortion $=47.2 \%$

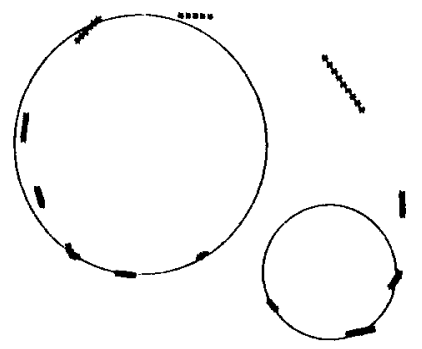

d) Rib Distortion $=0.08$

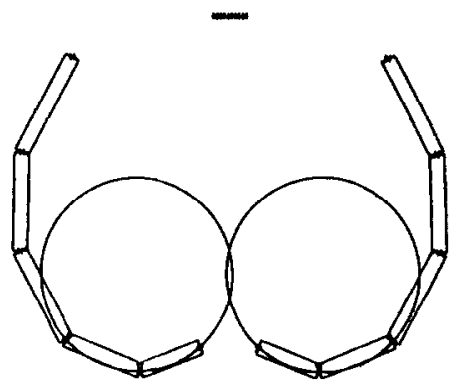

e) Rib Distortion $=4.4 \%$

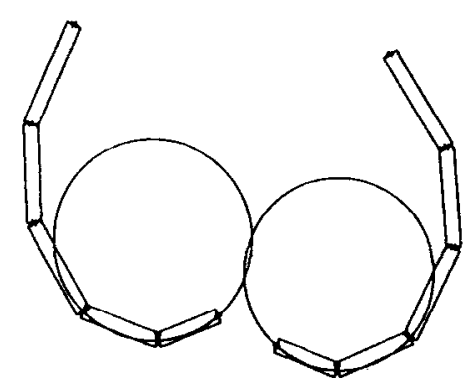

f) Rib Distortion $=5.48$

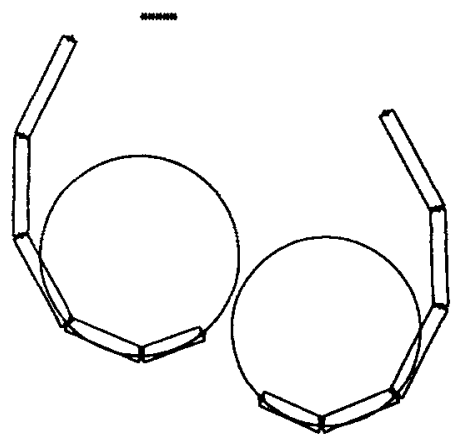

Fig. 4. View from superior of best-fit circles to rib posterior segments. (a-c) Computer-simulated CT images of the rib cage at T9 with vertebral bodies omitted for clarity: (a) normal rib cage $\left(R_{\text {concave }}=5.30 \mathrm{~cm}, R_{\text {convex }}\right.$ $=5.30 \mathrm{~cm}$ ); (b) rib cage from the $43^{\circ}$ scoliosis $\left(R_{\text {concave }}=5.70 \mathrm{~cm}, R_{\text {convex }}=5.05 \mathrm{~cm}\right) ;(\mathrm{c})$ rib cage from the $70^{\circ}$ scoliosis $\left(R_{\text {concave }}=8.62 \mathrm{~cm}, R_{\text {convex }}=4.55 \mathrm{~cm}\right)$. $(\mathrm{d}-f)$ Computer image of the apical rih pair projected onto its least-squares plane with vertebral bodies omitted for clarity: (d) normal rib cage $\left(R_{\text {concave }}=6.55 \mathrm{~cm}, R_{\text {conves }}\right.$ $=6.55 \mathrm{~cm})$; (e) rib cage from the $43^{\circ}$ scoliosis $\left(R_{\text {concave }}=6.78 \mathrm{~cm}, R_{\text {convex }}=6.48 \mathrm{~cm}\right)$; (f) rib cage from the $70^{\circ}$ scoliosis $\left(R_{\text {coneave }}=6.80 \mathrm{~cm}, R_{\text {convex }}=6.43 \mathrm{~cm}\right)$. The CT image clearly exaggregates the rib distortion. 
Table 1. Beam element basic stiffness values

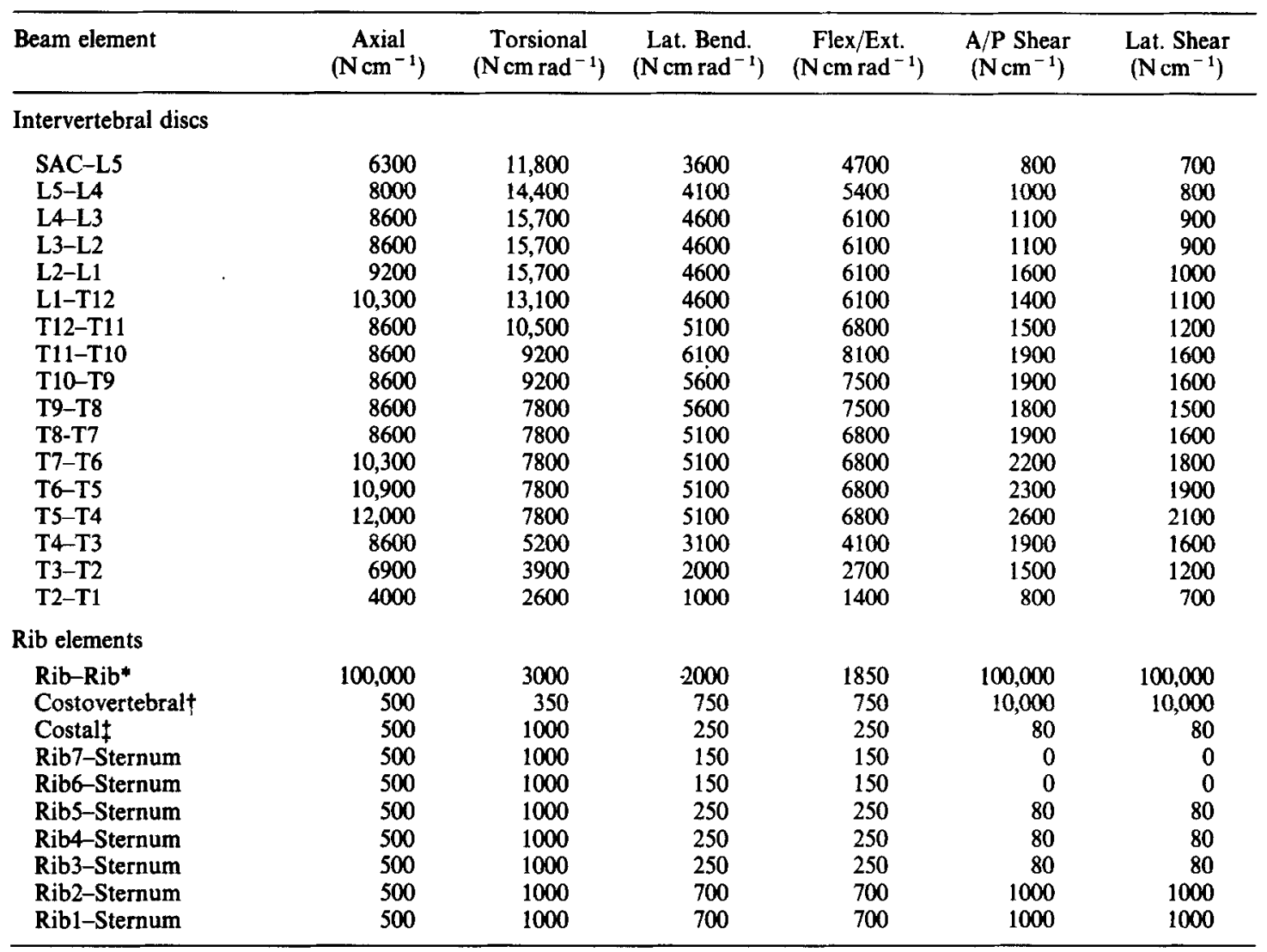

*Beam elements interconnecting all cylinders at all levels.

tAll levels.

†Rib7-Rib8, Rib8-Rib9, Rib9-Rib10 (represents costal cartilage tissue).

Note. All values except Rib-Rib beam elements are from Andriacchi et al. (1974).

$100 \mathrm{~N}$ lateral-squeezing load applied to the ribs at the xiphoid process level produced a lateral rib cage diametrical change of $1.86 \mathrm{~cm}$ and an anterior/posterior diametrical change of $0.23 \mathrm{~cm}$. These model-predicted values are in better agreement with the results in vivo reported by Agostoni et al. (1966) than those predicted by the earlier rigid-rib model (Fig. 6).

The model rib cage response to a $100 \mathrm{~N}$ load concentrated at the xiphoid resulted in a $0.3 \mathrm{~cm}$ anterior displacement of the sternum with negligible $\left(0.8^{\circ}\right)$ sternal rotation in the sagittal plane (Fig. 7). If the sternum is considered as a rigid body and the supporting rib cage is viewed as a single translational spring, the model predicts that this supporting spring would have a stiffness of $333.3 \mathrm{~N} \mathrm{~cm}^{-1}$. The experiments of Patrick et al. (1965) using embalmed cadavers and Nahum et al. (1970) using fresh cadavers reported similar stiffness values.

The model-predicted rotations in response to an imposed $51^{\circ}$ axial rotation at the $\mathrm{T} 1$ vertebra were similar to those of Lovett (1905) (Fig. 8).

These three simulations indicated that the present model was able to predict assembled rib cage mechan- ical behavior reasonably well in a variety of loading situations.

\section{Rib deformities in scoliosis}

All rib deformity parameters except the total thoracic volume increased when the scoliosis Cobb angle was increased (Table 2). Rib cage axial rotation increased in proportion to apical vertebral rotation. The total rib cage volume remained essentially constant at 8.141 . However, the distribution of that volume changed as the spine scoliosis increased from 0 to $70^{\circ}$. In the normal spine the thoracic volume was equally distributed on the convex and concave sides. As the scoliosis increased, the concave side volume increased while the convex side volume decreased, and in the $70^{\circ}$ curve, the concave side volume was $5 \%(0.201)$ larger than the convex side volume.

Rib distortion was clearly dependent on the view examined. For the $70^{\circ}$ scoliosis the rib distortion was $5.4 \%$ in the best-fit plane of the rib and $47.2 \%$ in the simulated CT scan plane. This indicates that a small asymmetry between rib pairs can produce a large apparent rib distortion in $\mathrm{CT}$ views. 


\section{Model Deflection vs. Experimental Mean Deflection}
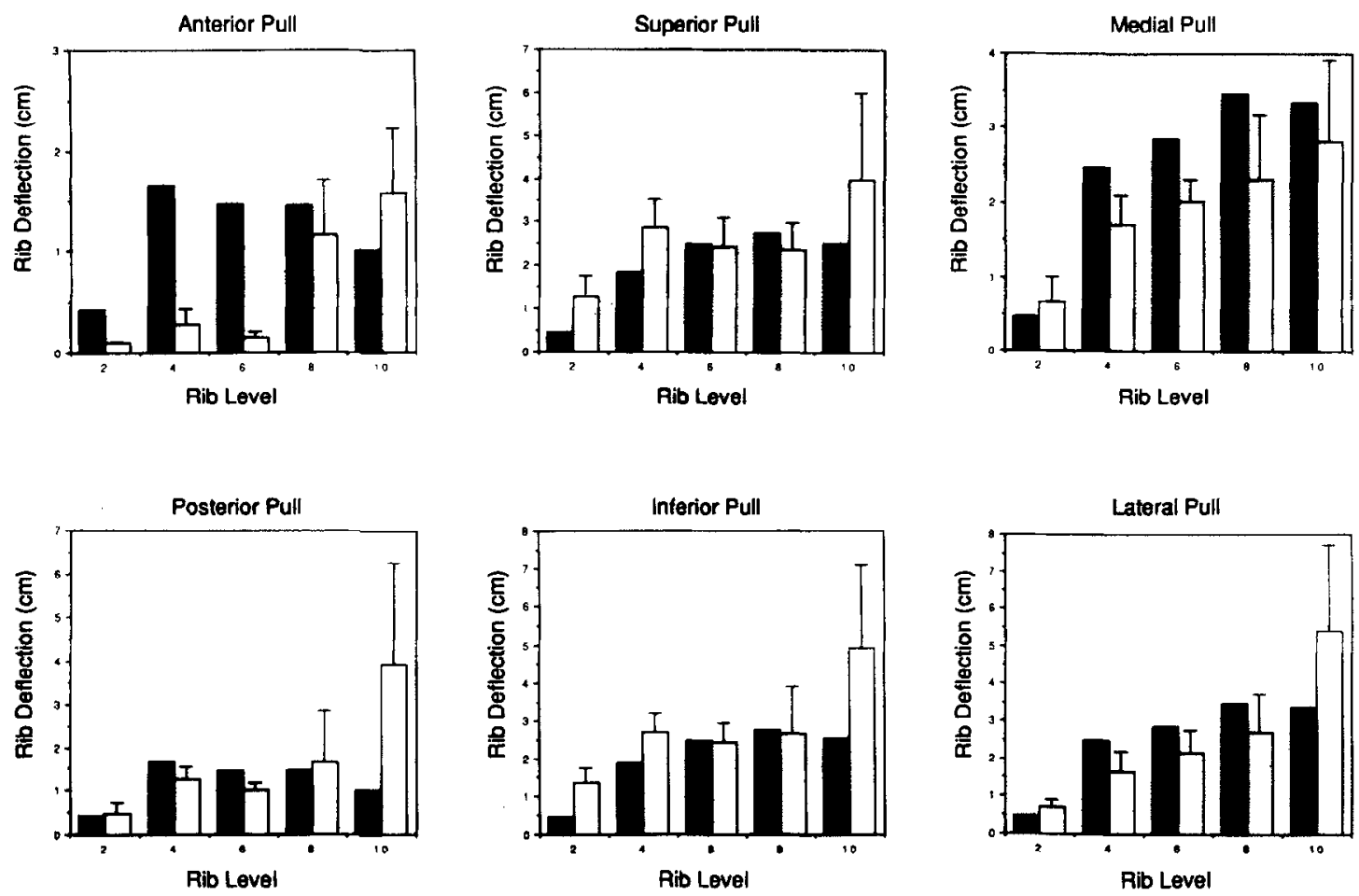

Model Deflection

Schultz et al. (1974) Mean

ABS910717/02

Fig. 5. Deflections of individual deformable ribs in response to $7.4 \mathrm{~N}$ loads. Model-predicted responses are compared to experimental measurements (Schultz et al., 1974) in response to loads applied in the six different directions. Measured mean values and standard deviations are shown.

For the normal spine the line formed by the intersection of the least-squares plane of the rib at T9 with a frontal plane was oriented at $35^{\circ}$ to the horizontal (Table 2). The line formed by the intersection of the rib plane with the sagittal plane was oriented at $27^{\circ}$ to the horizontal. Both scolioses produced asymmetric bestfit planes of the ribs as viewed frontally. The line formed by the intersection of the least-squares plane of the right rib plane at T9 with the frontal plane in the $70^{\circ}$ curve subtended an angle of $31^{\circ}$ to the horizontal, while the left rib plane subtended an angle of $38^{\circ}$. The lateral orientation of the best-fit rib plane was approximately the same for the normal and scoliotic spines.

\section{DISCUSSION}

Our primary motivation for the development of this deformable-rib model was to have a tool for studies of rib cage deformities in scoliosis. The results presented here show that the model developed adequately predicts the experimental measurements of whole rib cage behavior both in vivo and in vitro, and is also useful to quantify parameters measuring rib cage deformities.
This study addressed a number of issues concerning both how to model the rib cage and then how to use the model to study the complex rib cage deformities associated with scoliosis. In answer to the questions posed in the Introduction, we have the following results.

(1) The stiffnesses of beam elements needed to make model individual behavior agree with real rib behavior. We found that the three bending stiffnesses of each beam element are primarily responsible for rib mechanical behavior. The axial stiffnesses of the beam elements could be decreased by two orders of magnitude with negligible change in rib mechanical behavior. This supported our assumption that the shear and axial displacements are negligible. All beam elements interconnecting the rib cylinders were assigned the same set of six stiffnesses, yet the behavior of individual ribs at all levels agreed with the experimental observations (Schultz et al., 1974). The variation in stiffness between ribs from different anatomical leveis is primarily a result of rib gross shape rather than either bone cross-sectional geometry or bone material properties. 


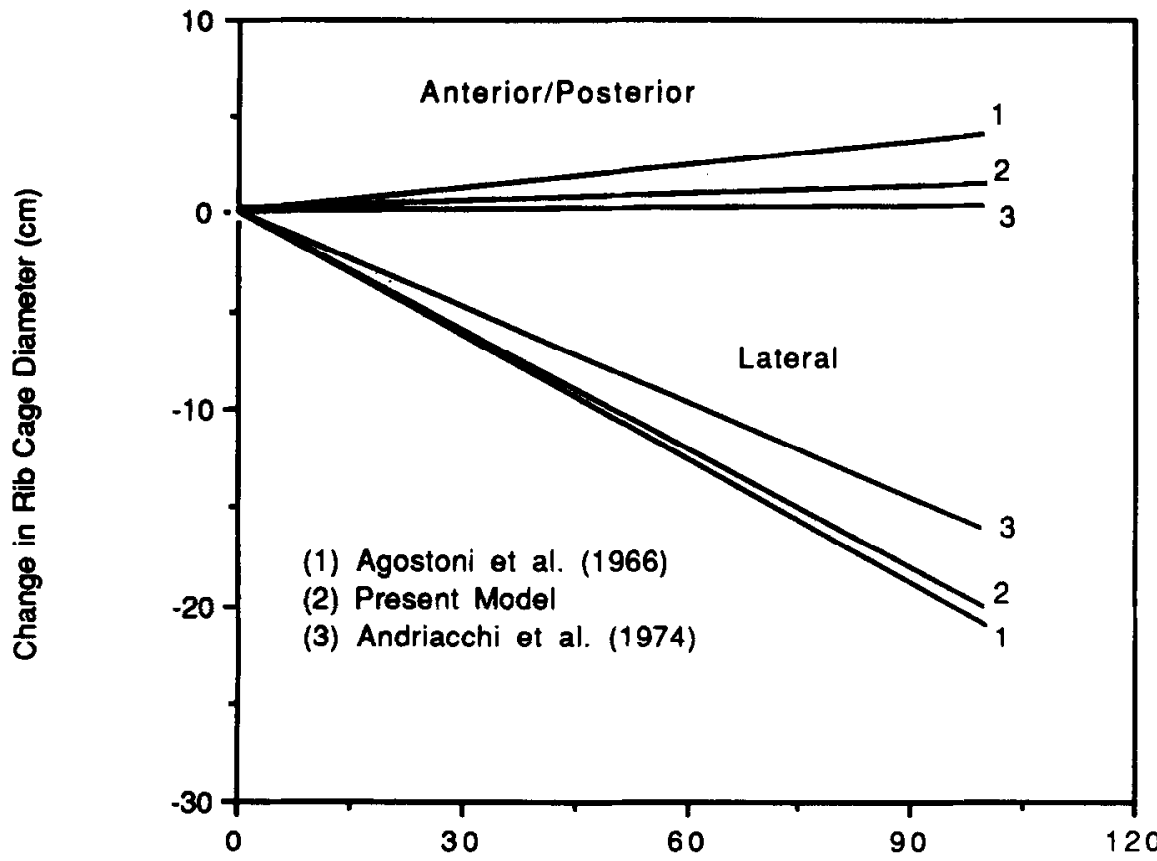

Lateral Load Applied to Rib Cage (N)

Fig. 6. Force-deflection responses of the assembled model rib cage. Model-predicted lateral and anteroposterior rib cage diameters in response to a uniform squeezing load are compared to the experimental measurements (Agostoni et al., 1966) and the earlier model predictions (Andriacchi et al., 1974).

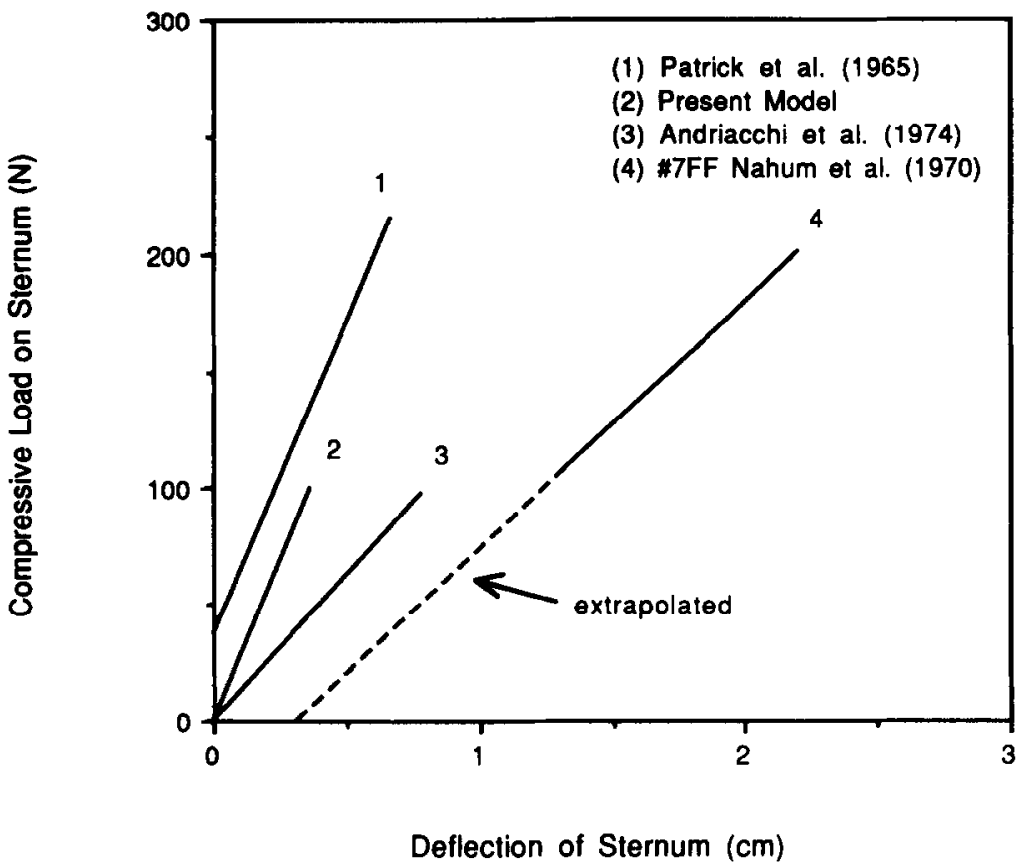

Fig. 7. Force-deflection responses of the assembled model rib cage. Model-predicted sternal deflection in response to a compressive load applied to the sternum is compared to the experimental findings (Nahum $e t$ al., 1970; Patrick et al., 1965) and the earlier model predictions (Andriacchi et al., 1974). 


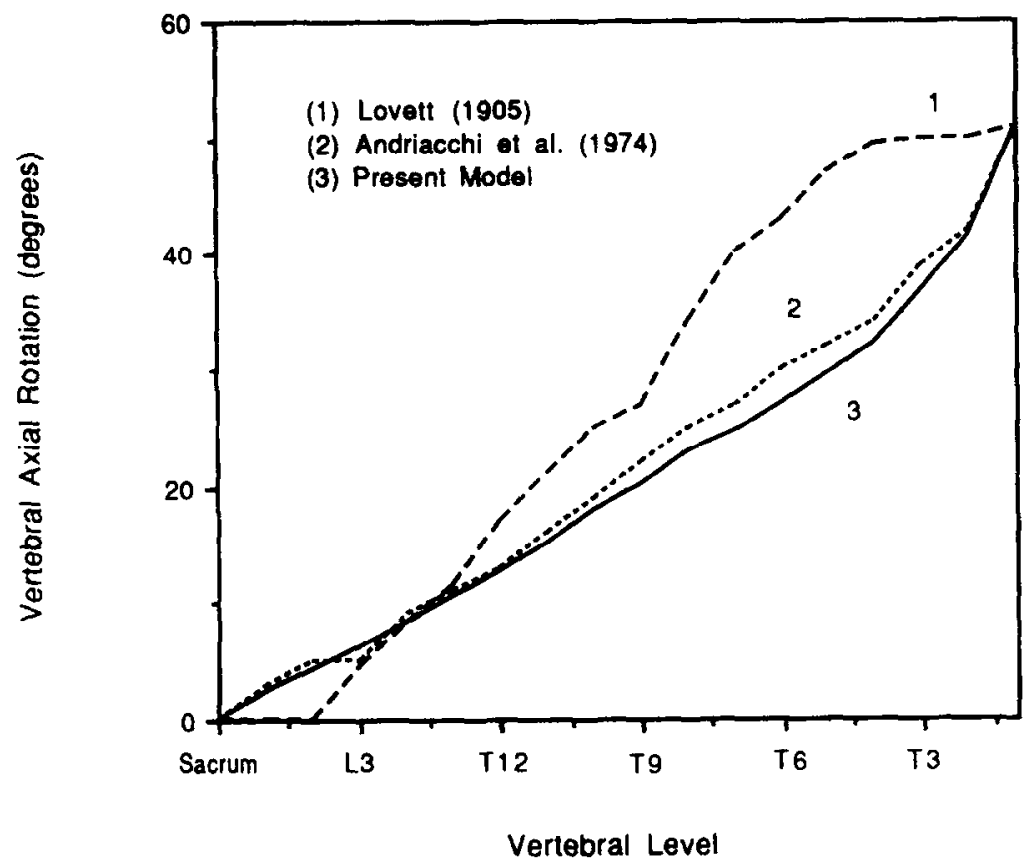

Fig. 8. Assembled model rib cage response to an axial rotation applied to the $\mathrm{T} 1$ vertebra compared to the experimental findings (Lovett, 1905) and the earlier model predictions (Andriacchi et al., 1974).

Table 2. Spine and rib cage morphology

\begin{tabular}{llll}
\hline $\begin{array}{l}\text { Input spine configuration } \\
\text { Mid-thoracic (T3-L3) scoliosis Cobb angle }\left(^{\circ}\right)\end{array}$ & 0 & 43 & 70 \\
Apical vertebral (T9) axial rotation $\left(^{\circ}\right)$ & 0 & 20 & 45 \\
\hline Resulting data at the apical rib level & & & \\
\hline Rib cage lateral offset (cm) & 0.0 & 1.87 & 2.37 \\
Rib cage axial rotation ( $\left.{ }^{\circ}\right)$ & 0.0 & 6.0 & 15.0 \\
Rib cage volume (1) & 8.14 & 8.16 & 8.13 \\
Rib cage volume ratio (concave/convex) & 1.00 & 1.03 & 1.05 \\
Rib distortion: best-fit plane (\%) & 0.0 & 4.4 & 5.4 \\
Rib distortion: CT Scan (\%) & 0.0 & 11.4 & 47.2 \\
Rib plane frontal inclination $\mathrm{L} / \mathrm{R}\left(^{\circ}\right)^{*}$ & $35 / 35$ & $37 / 32$ & $38 / 31$ \\
Rib plane sagittal inclination $\mathrm{L} / \mathrm{R}\left({ }^{\circ}\right)^{*}$ & $27 / 27$ & $28 / 27$ & $28 / 27$ \\
\hline
\end{tabular}

*Anglc formed between a horizontal surface and the line of intersection between the best-fit rib plane and the indicated anatomical plane.

The model represents each rib as a collection of five rigid cylinders. This choice was somewhat arbitrary. It holds the model degrees of freedom to 714 while reasonably mimicking real rib mechanical behavior. Although the five-cylinder representation limits the range of shapes the deformed rib can assume, these shapes exhibit all major clinical features of a scoliotic rib deformity (Fig. 9). The incremental accuracy gained by use of a larger number of cylinders would not seem to justify the increased computation time.

(2) The agreement of model and real overall cage behaviors in structurally normal trunks when model and real individual rib behaviors agree. This rib cage model produced better agreement with the real rib cage deflections reported by Patrick et al. (1965) and Agostoni et al. (1966) when compared to the earlier model. This better representation of rib deformation results from the ability of the ribs to deform under the loading conditions imposed. Additionally, Kroell et al. (1965) reported that if the thorax is viewed as a single translational spring, then its stiffness in response to sternal compression would be in the range of $325-800 \mathrm{~N} \mathrm{~cm}^{-1}$. Model findings are in agreement.

Although the model is more compliant than the earlier model in response to lateral loading, it is stiffer in response to sternal loading. The increase in stiffness when the model is sternally loaded is due to the addition of the pelvosternal springs. When these 


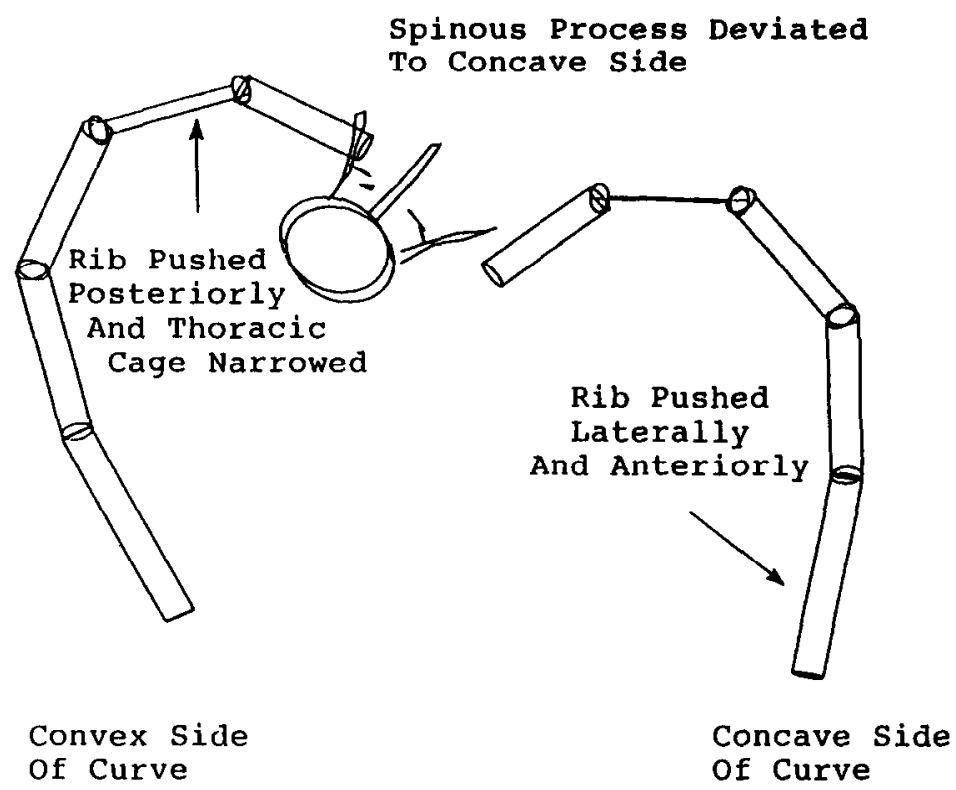

Fig. 9. Model agreement with the clinical characteristics of rib distortion in thoracic scoliosis (adopted from Keim and Netter, 1972).

springs were removed, the model response to sternal loading was found to be less stiff than the earlier rigidrib model (a deflection of $0.98 \mathrm{~cm}$ under an $80 \mathrm{~N}$ sternal load).

The model simulation results reported by Lovett (1905) refer to a single spine. We are unaware of more recent work of this nature. Our model does predict reasonable spinal torsional response to rotational loading compared to Lovett's results.

The model-predicted orientations of the $\mathrm{T} 9 \mathrm{rib}$ planes in the structurally normal trunk are in agreement with the reported data on normal human rib geometry in vivo (Wilson et al., 1987; Dansereau and Stokes, 1988; Stokes et al., 1989).

geometric linearity and rigid bony structures seemed adequate. These assumptions yielded reasonable computation time and a sufficiently representative model. Our model does not include the effects of muscles that attach to the spine and rib cage, but these can be incorporated using the procedures of Takashima $e t a l$. (1979). Despite these limitations, our model seems a useful tool to investigate the mechanics of rib cage deformation.

(3) Rib cage lateral offset, rib cage axial rotation, rib cage volume and rib distortion as quantitative measures of rib cage deformity in scoliosis. Rib cage axial rotations and rib cage lateral offsets have been previously proposed as measures of rib cage deformity (Thulbourne and Gillespie, 1976; Benson et al., 1977; Aaro and Dahlborn, 1981b; Pun et al., 1987; Jefferson et al., 1988). These measures seem to be linearly related to spine rotations and lateral offsets (Closkey and Schultz, 1991). However, individual rib distortions have not yet been reported.
When a rib in a mild or moderate scoliosis is viewed in its least-squares plane, the only deformity seen is the rather small distortion of the rib. Nevertheless, this yields a large apparent rib distortion when viewed in a simulated CT scan, apparently because of the effects of superposition of the ribs at various levels with different amounts of rib axial rotation.

Breathing should have little effect on these measurements. Jordanoglou (1969) reported that during tidal breathing the ribs are not elastically deformed to any measurable degree and that they undergo a single-axis rotation about the rib neck. He found this to be true in both normal and kyphoscoliotic rib cages. The amount of rib rotation about a single axis at the rib

\section{was $1.5^{\circ}$.}

(4) Rib cage volume differences between structurally normal and deformed scoliotic spines. The calculated rib cage volume of 8.141 is in agreement with the reported literature on total thoracic volume (Rodgers and Tannen, 1983). Our initial studies found that although the total rib cage volume changes little, the volume distribution becomes asymmetric. This suggests that factors other than cage total volume are involved in the cardiopulmonary complications often associated with scoliosis.

(5) Rib distortions in scoliosis. The CT image of the rib cage probably indicates well the cosmetic deformity of the thorax. Cundy et al. (1990) were among the first to use the CT image to assess thoracic cosmetic deformity. However, it is important to understand how rib deformity in the CT scan and the best-fit planes relate. Using this new model's ability to quantify changes in rib shape both in best-fit and CT scan planes, we found that individual scoliotic ribs may be 
much less severely deformed than they appear to be in CT images. We have provided simple quantitative measures of rib deformity in both planes in terms of percentage rib distortion.

Acknowledgements-Support of NIH Grant AR 33948 and the Vennema Endowment for this research is gratefully acknowledged.

\section{REFERENCES}

Aaro, S. and Dahlborn, M. (1981a) The longitudinal axis rotation of the apical vertebra, the vertebral, spinal, and rib cage deformity in idopathic scoliosis studied by computer tomography. Spine 6, 567-572.

Aaro, S. and Dahlborn, M. (1981b) Estimation of vertebral rotation and the spinal and rib cage deformity in scoliosis by computer tomography. Spine 6, 460-467.

Aaro, S. and Dahlborn, M. (1982) The cffect of Harrington instrumentation on the longitudinal axis rotation of the apical vertebra and on the spinal and rib cage deformity in idiopathic scoliosis studied by computer tomography. Spine 7, 456-462.

Aaro, S. and Ohlund, C. (1984) Scoliosis and pulmonary function. Spine 9, 220-222.

Agostoni, E., Mognoni, P., Torri, G. and Miserocchi, G. (1966) Forces deforming the rib cage. Resp. Physiol. 2, 105-117.

Andriacchi, T., Schultz, A., Belytschko, T. and Galante, J. (1974) A model for studies of the mechanical interactions between the human spine and rib cage. J. Biomechanics 7 , 497-507.

Benson, R. B., DeWald, R. L. and Schultz, A. B. (1977) Harrington rod distraction instrumentation its effect on vertebral rotation and thoracic compensation. Clin. Orthop. Rel. Res. 125, 40-44.

Closkey, R. F. and Schultz, A. B. (1992) Rib cage deformities in scoliosis: biomechanical analyses (submitted).

Cundy, P. J., Paterson, D. C., Hillier, T. M., Sutherland, A. D., Stephen, J. P. and Foster, B. K. (1990) Cotrel-Dubousset instrumentation and vertebral rotation in adolescent idiopathic scoliosis. J. Bone Jt Surg. 72B, 670-674.

Dansereau, J. and Stokes, I. A. F. (1988) Measurements of the three-dimensional shape of the rib cage. $J$. Biomechanics 21, 893-901.

Feiss, H. O. (1906) The mechanics of lateral curvature. Am. J. Orthop. Surg. 4, 37-68.

Gaines, R. W., Mercer McKinley, L. and Leatherman, K. D. (1981) Effect of the Harrington compression system on the correction of the rib hump in spinal instrumentation for idiopathic scoliosis. Spine 6, 489-493.

Gazioglu, K., Goldstein, L. A., Femi-Pearse, D. and Yu, P. N. (1968) Pulmonary function in idiopathic scoliosis. J. Bone Jt Surg. 50A, 1391-1399.

Jefferson, R. J., Weisz, I., Turner-Smith, A. R., Harris, J. D. and Houghton, G. R. (1988) Scoliosis surgery and its effect on back shape. J. Bone $J t$ Surg. 70B, 261-266.

Jordanoglou, J. (1969) Rib movement in health, kyphoscoliosis, and ankylosing spondylitis. Thorax 24, 407-414.

Keim, H. A. and Netter, F. H. (1972) Scoliosis. Clinical Symposia 24 (Edited by Roberts, R. H.), pp. 1-32. CIBA, Summitt, NJ.

Kroell, C. K., Patrick, L. M. and Mertz, H. J. (1965) Forces on the Human body in simulated crashes. G. M. Res. Publ.
No. GME-506, General Motors Corp.

Kumano, K. and Tsuyama, N. (1985) Pulmonary function before and after surgical correction of scoliosis. $J$. Bone $J t$ Surg. 64A, 242-249.

Lindh, M. and Bjure, J. (1975) Lung volumes in scoliosis before and after correction by the Harrington instrumentation method. Acta orthop scand. 46, 934-948.

Lovett, R. W. (1905) The mechanism of the normal spine and its relation to scoliosis. Bos. med. Surg. J. 153, 349-358.

Nahum, A. M., Gadd, C. W., Schneider, D. C. and Kroell, C. K. (1970) Deflection of the human thorax under sternal impact. In Proceedings of Inter. Auto Safety Conf., Detroit and Brussels, pp. 779-807.

Ogilvie, J. W. and Schendel, M. J. (1988) Calculated thoracic volume as related to parameters of scoliosis correction. Spine 13, 39-42.

Patrick, L., Kroell, C. and Mertz, H. (1965) Forces on the human body in simulated crashes. In Proceedings of 9 th Stapp Car Crash Conf., pp. 237-259.

Pecorelli, F., Grassi, V., Ferrini, L. and Todisco, T. (1983) Changes in respiratory function in disorders of the thoracic cage. Ital. J. Orthop. Trauma 9, 75-89.

Pun, W. K., Luk, K. D. K., Lee, W. and Leong, J. C. Y. (1987) A simple method to estimate the rib hump in scoliosis. Spine 12, 342-345.

Roberts, S. B. and Chen, P. H. (1970) Elastostatic analysis of the human thoracic skeleton. J. Biomechanics 3, 527-545.

Rodgers, R. P. C. and Tannen, R. (1983) Rapid and accurate determination of total lung capacity (TLC) from routine chest radiograms using a programmable hand-held calculator. Comput. Biol. Med. 13, 125-140.

Schultz, A. B., Belytschko, T. P., Andriacchi, T. P. and Galante, J. O. (1973) Analog forces in the human spine: mechanical properties and motion segment behavior. $J$. Biomechanics 6, 373-383.

Schultz, A. B., Benson, D. R. and Hirsch, C. (1974) Forcedeformation properties of human ribs. J. Biomechanics 7 , 303-309.

Smith, R. M., Hamlin, G. W. and Dickson, R. A. (1991) Respiratory deficiency in experimental idiopathic scoliosis. Spine 16, 94-99.

Stokes, I. A. F. (1989) Axial rotation component of thoracic scoliosis. J. orthop. Res. 7, 702-708.

Stokes, I. A. F., Dansereau, J. and Moreland, M.S . (1989) Rib cage asymmetry in idiopathic scoliosis. J. orthop. Res. 7 , 599-606.

Takashima, S. T., Singh, S. P., Haderspeck, K. A. and Schultz, A. B. (1979) A model for semi-quantitative studies of muscle actions. J. Biomechanics 12, 929-939.

Thulbourne, T. and Gillespie, R. (1976) The rib hump in idiopathic scoliosis. J. Bone Jt Surg. 58B, 64-71.

Weatherly, C. R., Draycott V., O'Brien, J. F., Benson, D. R., Gopalakrishnan, K. C., Evans, J. H. and O'Brien, J. P. (1987) The rib cage deformity in adolescent idiopathic scoliosis. J. Bone Jt Surg. 69B, 179-182.

Wilson, T. A., Rehder, K., Krayer, S., Hoffman, E. A., Whitney, C. G. and Rodarte, J. R. (1987) Geometry and respiratory displacement of human ribs. J. appl. Physiol. 62, 1872-1877.

Winter, R. B., Lovell, W. W. and Moe, J. H. (1975) Excessive thoracic lordosis and loss of pulmonary function in patients with idiopathic scoliosis. J. Bone Jt Surg. 57A, 972-977.

Yamada, H. (1970) Strength of Biological Materials (Edited by Evans, F. G.), p. 97. Williams and Wilkins, Baltimore, MD. 